\title{
The Influence of Artificial Ventilation on Heart Rate Variability in Very Preterm Infants
}

\author{
CONNY M. A. vaN RAVENSWAAIJ-ARTS, JEROEN C. W. HOPMAN, LOUIS A. A. KOLLÉE, \\ GERARD B. A. STOELINGA, AND HERMAN P. VAN GEIJN \\ Department of Paediatrics [C.M.A.V., J.C.W.H., L.A.A.K., G.B.A.S.], University Hospital, Nijmegen, and \\ Department of Obstetrics and Gynaecology [H.P.V.], Free University Hospital, Amsterdam, \\ The Netherlands
}

\begin{tabular}{|c|c|}
\hline $\begin{array}{l}\text { To study the influence of artificial ventilation rate on neonatal } \\
\text { heart rate variability (HRV), ECG and respiratory impedance } \\
\text { curves were recorded four times a day in } 20 \text { preterm infants ( }<33 \\
\text { wk) during the first } 3 \mathrm{~d} \text { after birth while the infants were } \\
\text { ventilated at a wide range of ventilator rates. The contents of } \\
\text { selected frequency bands within the R-R interval power spectrum } \\
\text { were calculated for 3-min periods. Respiratory distress syndrome } \\
\text { severity was assessed at each measurement. Respiratory sinus } \\
\text { arrhythmia (RSA) induced by the ventilator appeared to mimic } \\
\text { spontaneous RSA. As in spontaneous respiration, the amount of } \\
\text { RSA (power in a frequency band around the respiratory rate) } \\
\text { increases as the ventilation rate decreases. This phenomenon is } \\
\text { most probably due to entrainment with baroreflex-related fluctu- } \\
\text { ations in the heart rate. Although the artificial ventilation rate } \\
\text { influences RSA and thus high-frequency HRV, an increase in }\end{array}$ & 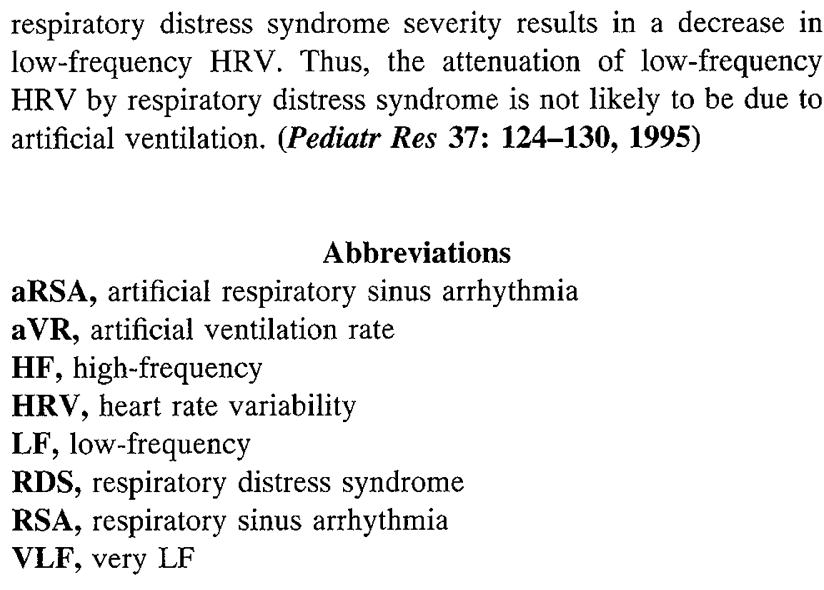 \\
\hline
\end{tabular}

HRV, i.e. the variation in beat-to-beat R-R interval length, is influenced by maturational (e.g. gestational age), physiologic (e.g. respiration), and clinical factors $(1,2)$. HRV can be assessed by spectral analysis to study the frequency-specific oscillations in the heart rate signal. In the newborn, the following heart rate oscillations may be present. HF oscillations have a frequency equal to the respiratory rate and are known as RSA. LF oscillations are due to intrinsic oscillations of the baroreceptor reflex loop with a frequency of $0.07 \mathrm{~Hz}$ (3). A second kind of LF oscillation may result from periodic fluctuations in respiratory depth or tidal volume (4). The last type of HRV features VLF oscillations with a frequency below 0.04 $\mathrm{Hz}$. These are ascribed to thermoregulation. HF oscillations are mediated by the parasympathetic system. LF and VLF oscillations are mediated by both the sympathetic and parasympathetic systems (5). Thus, studying neonatal HRV can give an insight into the maturation of the autonomic nervous system.

In newborns suffering from RDS, HRV appears to be attenuated $(2,6,7)$. With improvement of the neonatal condition,

Received October 14, 1993; accepted July 22, 1994.

Correspondence: Dr. C. M. A. van Ravenswaaij-Arts, Department of Paediatrics, University Hospital Nijmegen, P.O. Box 9101, 6500 HB Nijmegen, The Netherlands.
HRV returns to normal (2). Several explanations have been proposed for this reversible effect of RDS on HRV. For instance, it has been ascribed to transient depression of the medulla oblongata by hypercarbia (2). The possible influence of artificial ventilation as such on neonatal HRV has not been taken into account in studies regarding the influence of RDS. For the correct interpretation of HRV in infants with RDS, insight into the effect of artificial ventilation on HRV is needed. This influence cannot be studied by simply comparing HRV of spontaneously breathing infants with HRV of ventilated newborns, because these infants differ greatly, especially in clinical condition. An attempt has been made to compare HRV in preterm newborns before and after extubation (2). This comparison might also be biased, e.g. by clinical condition and postnatal age. To gain an insight into the influence of artificial ventilation on HRV in preterm newborns and to avoid the methodologic problems just mentioned, we investigated the influence of aVR on neonatal HRV. We studied whether the negative influence of RDS on the regulatory systems that contribute to HRV could be explained by the influence of artificial ventilation. We also compared the influence of aVR in ventilated newborns to the influence of spontaneous ventilation rate on neonatal HRV as obtained in otherwise comparable 
infants and reported previously (8). The mechanism by which artificial ventilation influences HRV is discussed.

\section{METHODS}

Subjects. Infants born at a gestational age of $33 \mathrm{wk}$ and less and admitted to the neonatal intensive care unit within $12 \mathrm{~h}$ of birth entered the study. Exclusion criteria were presence of congenital malformations and use of cardiovascular or sedative drugs by mother or infant. Sixty-nine infants were originally included in the study $(2,8)$. Twenty infants were appropriate for gestational age and artificially ventilated at five or more different frequencies during the study period from birth to a postnatal age of $72 \mathrm{~h}$. Patient characteristics are tabulated in Table 1.

The results obtained in the ventilated infants were compared with the influence of spontaneous breathing in 16 preterm newborns with no or only mild symptoms of RDS (median gestational age $31 \mathrm{wk}$, range 27-32 wk; median birth weight $1550 \mathrm{~g}$, range $1110-2280 \mathrm{~g}$ ), measured under the same conditions as the intubated infants and reported in a previous study (8).

Data acquisition. Heart rate, respiration, and motility were assessed at regular intervals (0930, 1330, 1700, and $2130 \mathrm{~h}$ ) until $72 \mathrm{~h}$ after birth. Recordings were obtained at least $1 \mathrm{~h}$ after the last intervention (e.g. feeding, endotracheal suctioning). Only measurements obtained during 3 -min periods with no or regular spontaneous respiration and no or only small movements (of hands or feet) were used in our study. In the 16 spontaneously breathing infants, these periods were classified as state coincidence 1 (quiet-like sleep) $(8,9)$.

$\mathrm{R}$ waves in the ECG were detected by the QRS detector of a cardiorespiratory monitor (HP 78801B, Hewlett-Packard Nederland BV, Amstelveen, The Netherlands). The subsequent flash pulses were timed with a resolution of $0.1 \mathrm{~ms}$ by a PDP-11/23 microcomputer (Digital Equipment BV, Utrecht, The Netherlands). The respiratory impedance curve (sampling rate $40 \mathrm{~Hz}$ ) was also stored on-line. Measurements were continued until three periods of $3 \mathrm{~min}$ in stable condition were marked by the observer. Thus, a maximum of 12 measurement series consisting of three 3 -min periods each could be obtained per infant.

Spectral indices of HRV. An artifact correction procedure corrected the arrays of R-R intervals for missed and spurious $\mathrm{R}$ waves and for supraventricular extrasystoles (1). The recording was rejected if more than five artifact repairs were made within a 3-min period. Calculation of the power spectrum was performed on the 3-min periods of R-R intervals as described previously (8). The latter procedure included interpolation (second-order polynomial) and resampling $(5.69 \mathrm{~Hz}$ offering 1024 data points) of the R-R intervals, followed by detrending (subtracting the best linear fit) and calculation of the autocovariance function. Smoothing of this function was achieved by applying a Tukey window, yielding again 1024 data points (10). Fast Fourier transformation of this autocovariance function gives the power spectrum of R-R intervals with a standardized resolution of $7.4 \mathrm{mHz}(10)$.

Table 1. Characteristics of infants studied

\begin{tabular}{|c|c|c|c|c|c|c|c|c|c|}
\hline Patient & Sex & GA (wk) & BW (g) & RDS* & Apgar $\dagger$ & mode & aVR $\ddagger$ & US & Follow-up§ \\
\hline 1 & F & 26 & 860 & II & 7 & Vag & $12-35$ & $\mathrm{~N}$ & 0 \\
\hline 2 & M & 27 & 1070 & I & 9 & Vag & $6-17$ & $\mathrm{~N}$ & 0 \\
\hline 3 & $M$ & 28 & 975 & II & 4 & Vag & $9-60$ & $\mathrm{~N}$ & 0 \\
\hline 4 & $\mathrm{~F}$ & 28 & 1000 & III & 7 & CS & $15-39$ & $\mathrm{~N}$ & 0 \\
\hline 5 & $\mathrm{~F}$ & 28 & 1040 & III & 8 & Vag & $15-64$ & PVL & 3 \\
\hline 6 & $\mathrm{~F}$ & 28 & 1125 & I & 5 & Vag & $3-41$ & IVH & 0 \\
\hline 7 & $\mathrm{~F}$ & 28 & 1160 & III & 6 & Vag & $22-39$ & $\mathrm{~N}$ & 0 \\
\hline 8 & M & 28 & 1250 & II & $?$ & Vag & $10-37$ & $\mathrm{~N}$ & 0 \\
\hline 9 & F & 28 & 1430 & II & 9 & Vag & $3-56$ & IVH & 2 \\
\hline 10 & F & 29 & 1280 & II & 7 & Vag & $7-42$ & $\mathrm{~N}$ & 0 \\
\hline 11 & $\mathrm{~F}$ & 29 & 1295 & II & 10 & $\mathrm{CS}$ & $14-50$ & $\mathrm{~N}$ & 0 \\
\hline 12 & M & 30 & 1110 & III & 8 & CS & $29-54$ & $\mathrm{~N}$ & 0 \\
\hline 13 & $\mathrm{~F}$ & 30 & 1415 & II & 10 & Vag & $3-21$ & $\mathrm{~N}$ & 0 \\
\hline 14 & $M$ & 30 & 1600 & II & 8 & Vag & $6-22$ & $\mathrm{~N}$ & 1,2 \\
\hline 15 & $\mathrm{~F}$ & 31 & 1650 & III & 8 & Vag & $22-58$ & $\mathrm{~N}$ & 0 \\
\hline 16 & M & 32 & 1500 & II & 5 & Vag & $46-63$ & $\mathrm{~N}$ & 0 \\
\hline 17 & $\mathrm{~F}$ & 32 & 1605 & II & 7 & Vag & $5-38$ & $\mathrm{~N}$ & 0 \\
\hline 18 & M & 32 & 1720 & II & $?$ & CS & $7-43$ & IVH & 0 \\
\hline 19 & $\mathrm{~F}$ & 32 & 1800 & II & 9 & CS & $7-51$ & $\mathrm{~N}$ & 0 \\
\hline 20 & M & 32 & 2060 & III & 8 & CS & $9-38$ & $\mathrm{~N}$ & 0 \\
\hline Median & & 29 & 1288 & II & 8 & & & & \\
\hline
\end{tabular}

GA, gestational age; BW, birth weight; Vag, vaginal delivery; CS, caesarean section; US, transfontanellar ultrasound scan; N, normal; IVH, intraventricular hemorrhage without ventriculomegaly or involvement of the cerebral parenchyma; PVL, periventricular leukomalacia.

* RDS, overall RDS score (see text).

$\uparrow$ Apgar score at 5 min after birth.

+ Range aVR (per min).

$\S$ Psychomotor development at the corrected age of 1 y postterm: 0 , unremarkable; 1 , mild hypertonia; 2 , mild developmental delay (less than 3 mo behind);

3 , died at the age of $44 \mathrm{~d}$ after birth due to bronchopulmonary dysplasia. 
To obtain spectral indices of HRV, the power $\left(\mathrm{ms}^{2}\right)$ in selected frequency bands was calculated. Most frequency bands chosen were based on the study of Baldzer et al. (11). These frequency bands correspond to VLF $(0.017-0.04 \mathrm{~Hz})$, LF (0.04-0.2 Hz), and HF (above $0.2 \mathrm{~Hz}$ ) oscillations (Fig. 1). We added a spectral index to quantify the influence of aVR more precisely. In the R-R interval power spectrum, the power in a spectral band of $0.4 \mathrm{~Hz}$ around the $\operatorname{aVR}(\mathrm{aVR} \pm 0.2 \mathrm{~Hz}=$ aRSA index) was calculated (Fig. 1).

Other parameters. For each 3-min period, the mean R-R interval length was calculated. The severity of RDS was scored at each measurement series as class I (mild), II (moderate), or III (severe), according to Korvenranta et al. (7). An overall RDS score was assigned to each infant based on the worst RDS score that was given at least three times (Table 1). In the 16 spontaneously breathing infants, median respiratory rate was
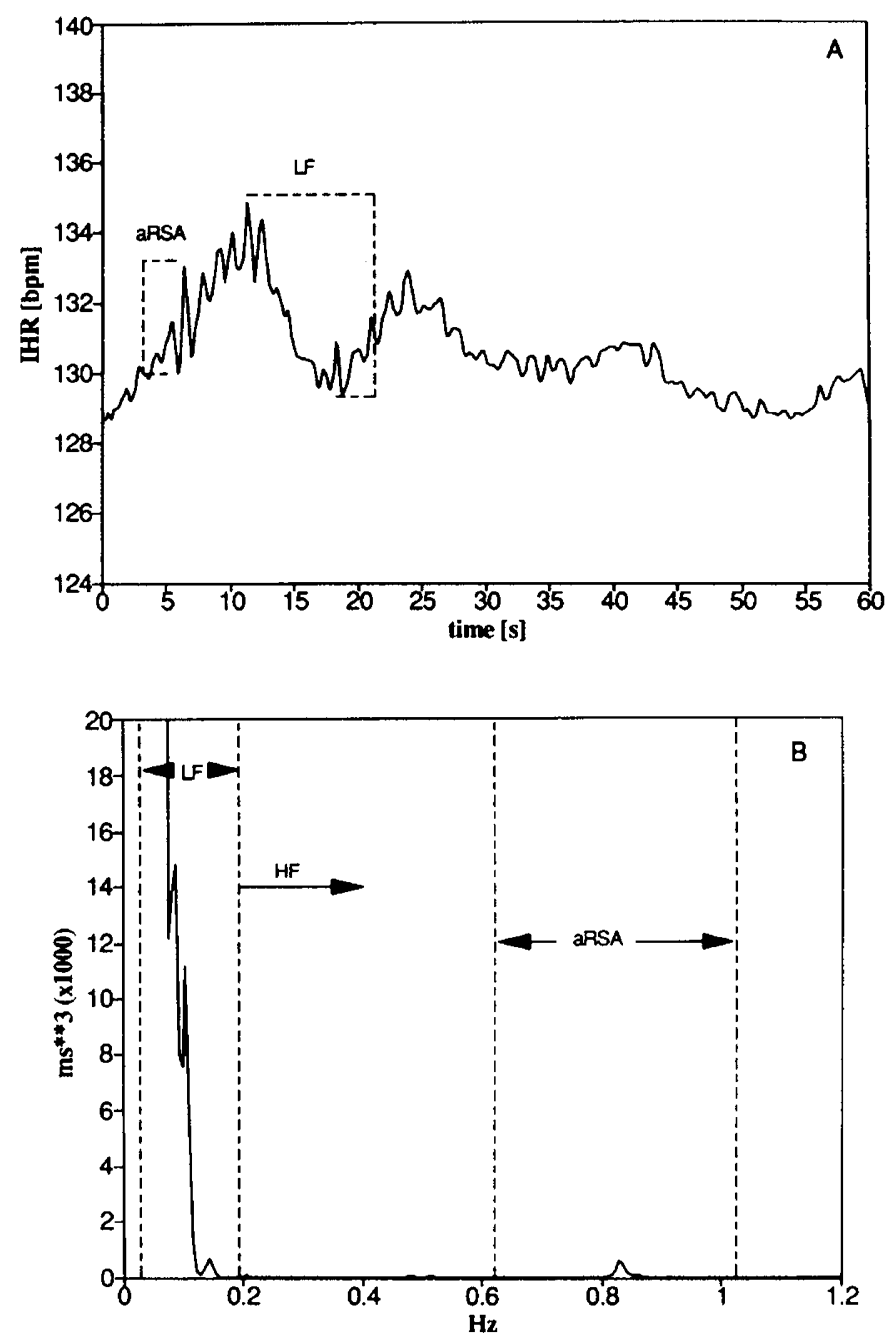

Figure 1. Spectral indices of HRV. Interpolated instantaneous heart rate $(I H R)$ trace $1(A)$ and $\mathrm{R}-\mathrm{R}$ interval power spectrum $(B)$ of patient 19 at the age of $12 \mathrm{~h}$ after birth. In the heart rate trace, HF fluctuations induced by the respirator (aRSA, see detail of same measurement in Fig. $2 A$ ) as well as LF fluctuations are visible. The following HRV indices are indicated in the power spectrum $(B)$ : LF $(0.04-0.2 \mathrm{~Hz}$ ), HF (above $0.2 \mathrm{~Hz}$ ), aRSA (ventilation rate \pm $0.2 \mathrm{~Hz}$ ). Each individual instantaneous heart rate value was calculated from the present R-R interval. Subsequently the array of instantaneous heart rate values was interpolated (second-order polynomial), resulting in a smooth curve. obtained by calculating the power spectrum of the respiratory impedance curve. The frequency below which $50 \%$ of the spectral power occurs represents median respiratory rate (8).

Statistics. From the 20 selected infants, 392 reliable 3 -min periods could be used for statistical analysis. To obtain normally distributed HRV indices (VLF, LF, HF, and aRSA), they were $\log$ transformed. The within-patient median values were calculated first to obtain independent observations.

The $t$ test was performed on within-patient (Spearman) correlation coefficients between aVR and the HRV indices. Stepwise regression analysis (12) with the HRV indices as dependent variables and R-R interval length, postnatal and gestational age, aVR, and RDS as independent variables was performed on the complete set of 3923 -min periods. Results were considered significant at the 0.05 level.

From the 16 control infants, 156 reliable 3 -min periods were obtained during state coincidence 1 ("quiet" sleep). The same stepwise regression analysis was performed in these infants (with median respiratory rate instead of aVR) (8).

Ethical approval. The study was approved by the ethical committee of the University Hospital Nijmegen and informed parental consent was obtained for each infant.

\section{RESULTS}

The most important patient characteristics are shown in Table 1. Examples of heart rate traces in spontaneously breathing and artificially ventilated infants are given in Figure 2.

To obtain an insight into the relationships between aVR and mean R-R interval length, the HRV indices, and postnatal age, within-patient Spearman correlation coefficients were calculated (Table 2). A significant negative correlation of aVR with LF HRV (mean $r=-0.28$, SEM 0.12) and aRSA (mean $r=$ -0.64 , SEM 0.08) was found. However, postnatal age was also negatively correlated with aVR (mean $r=-0.40$, SEM 0.16) and thus might be a confounder.

In Table 3, the median absolute HRV values as well as gestational and postnatal age of four aVR classes are given. An example of the influence of ventilation rate on the amount of aRSA is shown in Figure 3. To correct for confounders (RDS severity, postnatal and gestational age) the influence of aVR on the different HRV indices was studied using stepwise regression analysis. In this analysis, the HRV indices were the dependent variables and R-R interval length, gestational and postnatal age, RDS severity, and aVR were the independent variables. The results are summarized in Table 4. aVR appeared to influence aRSA and HF HRV. In contrast, the severity of RDS influenced lower frequency HRV. For comparison, the results of a former study concerning spontaneously breathing preterm infants are also shown in Table 4 (8). In these infants, RSA is defined as the power spectral band around the median spontaneous ventilation rate $\pm 0.2 \mathrm{~Hz}$. Although these infants are not wholly comparable to the ventilated infants (no or only mild RDS and higher median gestational age), a tentative comparison can be made. The influence of RDS on VLF HRV in ventilated infants was stronger than the influence of R-R interval length as found in spontaneously breathing preterm infants. The influence of aVR on HF HRV in 

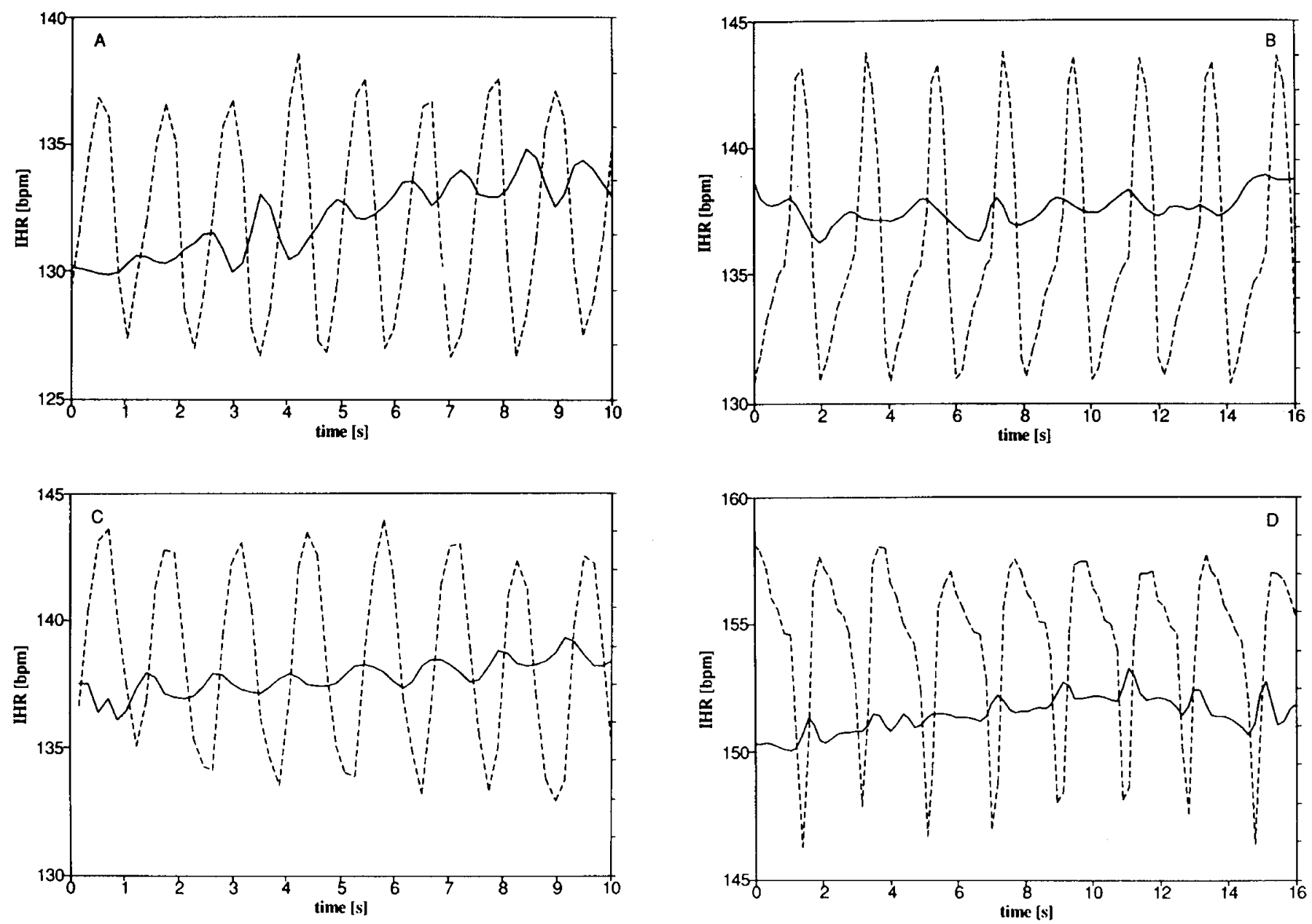

Figure 2. Influence of artificial and spontaneous breathing on the instantaneous heart rate (IHR). $A$ and $B$ are derived from patient 19 at the age of 12 and $36 \mathrm{~h}$ after birth, while ventilated at 0.83 and $0.50 \mathrm{~Hz}$, respectively. $C$ and $D$ have been derived from two spontaneously breathing infants at the age of 15 and $51 \mathrm{~h}$ after birth, with a breathing rate of 0.80 and $0.55 \mathrm{~Hz}$, respectively. The solid line indicates heart rate; the dashed line represents thoracic impedance. An ascending impedance curve refers to inspiration, a descending curve refers to expiration.

ventilated newborns was more pronounced than the influence of postnatal age as found in spontaneously breathing infants.

\section{DISCUSSION}

Inasmuch as we did not want to interfere with the routine care of the very preterm, intubated infants, it was not possible to investigate the influence of aVR on HRV by changing the frequency of the respirator on purpose. Therefore, we used data obtained from infants ventilated at a wide range of aVR. During the measurements, the newborns were ventilated at an

Table 2. Interindividual correlation coefficients of aVR with $R-R$ interval length, $H R V$, and postnatal age

\begin{tabular}{lcc}
\hline \multicolumn{3}{c}{ interval length, } \\
\hline Ventilation rate & $t$ Test \\
\hline MLF R-R & $-0.04(0.12)$ & NS \\
LF & $-0.20(0.13)$ & NS \\
HF & $-0.28(0.12)$ & $p<0.05$ \\
aRSA & $0.05(0.13)$ & NS \\
Age & $-0.64(0.08)$ & $p<0.001$ \\
\hline
\end{tabular}

Mean (SEM) of within-patient Spearman correlation coefficients of artificial ventilation rate with R-R interval length, log HRV indices (VLF, LF, HF, and aRSA), and postnatal age (age). $n=16$ infants. optimal aVR controlled by monitoring $\mathrm{PO}_{2}$ and $\mathrm{PCO}_{2}$ transcutaneously and by regularly obtained capillary blood gas values. The study population was limited to appropriate-forgestational-age infants to avoid the difference in HRV between appropriate- and small-for-gestational-age infants (1). Only measurements obtained during periods with no or regular spontaneous respiration and no or only small body movements were used to exclude 3-min periods with forced breathing against the respirator and to make measurements more comparable. Also, measurements of the control population were obtained during quiet sleep. During this sleep state, respiration is regular and more closely coupled to cardiac activity. During active sleep, not only a diminished central coupling of cardiac and respiratory related activity exists, but also paradox inward rib cage motion due to decreased postural tone in combination with high neonatal chest wall compliance can be observed (13). Thus, 3-min periods obtained during active sleep are less suitable to compare the influence of artificial and spontaneous ventilation on neonatal HRV.

As stated in the introduction, three main heart rate oscillations can be found in neonatal heart rate: HF oscillations coupled with respiratory activity, LF oscillations due to intrin- 
Table 3. Influence of $a V R$ on $H R V$

\begin{tabular}{lcccc}
\hline aVR (Hz) & $\leq 0.2$ & $0.2 \leq 0.4$ & $0.4 \leq 0.6$ & $>0.6$ \\
\hline$n$ & 13 & 17 & 13 & 15 \\
GA (wk) & 29 & 29 & 28 & 29 \\
& $(28-32)$ & $(28-31)$ & $(28-31)$ & $(28-32)$ \\
Age (h) & 35 & 33 & 32 & 14 \\
& $(24-39)$ & $(18-50)$ & $(17-60)$ & $(7-41)$ \\
aVR (Hz) & 0.14 & 0.30 & 0.51 & 0.76 \\
& $(0.13-0.15)$ & $(0.28-0.33)$ & $(0.48-0.56)$ & $(0.64-0.87)$ \\
R-R (ms) & 402 & 419 & 403 & 395 \\
& $(397-431)$ & $(394-432)$ & $(385-432)$ & $(385-427)$ \\
VLF & 9.70 & 10.1 & 4.06 & 5.89 \\
& $(2.70-37.9)$ & $(2.07-23.3)$ & $(2.11-9.15)$ & $(0.66-32.8)$ \\
LF & 3.45 & 3.23 & 2.77 & 1.12 \\
& $(1.03-10.4)$ & $(1.06-9.87)$ & $(0.57-4.58)$ & $(0.079-5.50)$ \\
HF & 0.042 & 0.044 & 0.015 & 0.005 \\
& $(0.005-0.15)$ & $(0.013-0.10)$ & $(0.005-0.049)$ & $(0.003-0.046)$ \\
aRSA & 18.4 & 0.36 & 0.014 & 0.003 \\
& $(5.33-119)$ & $(0.050-3.40)$ & $(0.003-0.039)$ & $(0.001-0.045)$ \\
\hline
\end{tabular}

Median (interquartile ranges) of GA and postnatal age, aVR, R-R interval length (R-R), and absolute HRV indices $\left[\times 10^{6} \mathrm{~ms}^{2}\right]$ (VLF, LF, HF, and aRSA) in four arbitrary ventilation rate classes.

sic oscillations in the vasomotor part of the baroreflex loop, and VLF oscillations due to fluctuations in peripheral vascular resistance (thermoregulation). The amplitude of the heart rate oscillations is determined by the strength of the perturbation (e.g. tidal volume) and the gain of the regulatory system (e.g. the autonomic nervous system or baroreflex). The frequency of the heart rate oscillations is determined by the frequency of the perturbation (e.g. ventilation rate) and the delay of the system. The latter explains why the baroreflex-related heart rate oscillations have a frequency of $0.1 \mathrm{~Hz}$ in adults and $0.07 \mathrm{~Hz}$ in newborns, i.e. the baroreflex has a longer time delay in newborns (3).

The increase in heart rate during spontaneous inspiration is due to inhibition of the vagal tone and concomitant attenuation of the baroreceptor reflex. This inspiratory inhibition has been ascribed to several mechanisms including the Bainbridge reflex, lung mechanoreceptor activity, and a central interaction between the respiratory and cardiovascular centers (14). The result is a fluctuation in heart rate with a frequency equal to the breathing rate (RSA). In the adult, RSA is augmented at lower breathing rates (15) due to entrainment with the $0.1-\mathrm{Hz}$ baroreflex-related heart rate oscillations (16). Thus, RSA is maximal at a breathing rate of six breaths per $\min ($ i.e. $0.1 \mathrm{~Hz})$ in adults. The decreased baroreflex sensitivity normally found during active inspiration has no equivalent during assisted breathing in healthy adult volunteers with voluntary suppression of the nervous inspiratory activity (17). Thus, the central interaction between respiratory and cardiovascular centers is not detectable during involuntary breathing. Yli-Hankala et al. (18) described a reversed RSA in artificially ventilated adults during anesthesia. They found a heart rate decrease during mechanical lung inflation in contrast to the centrally initiated increase in heart rate related to spontaneous inspiration. The artificially induced RSA may be due to changes in intrathoracic pressure that activate the baroreflex (through a direct transmural effect on the aortic baroreceptors) or the Bainbridge reflex (negative intrathoracic pressure during expiration instead of inspiration).
In Figure 2, examples of the respiratory impedance curve and instantaneous heart rate trace during artificial and spontaneous ventilation in our patients are shown. Thoracic impedance measurements reflect the gas:fluid ratio of the chest and give reliable information about the ventilation rate. In quiet newborns, the respiratory thoracic impedance changes are mainly due to changes in air volume within the chest (19). The initial increase in impedance with inspiration showed a delay of approximately $1 \mathrm{~s}(0.5-1.2 \mathrm{~s})$ compared with the change in expired $\mathrm{CO}_{2}$ and in thoracic circumference as measured by a mercury strain gauge (our unpublished observations). The magnitude of this delay may change per measurement, depending on, for example, the shape of the thorax and placement of the electrodes. In newborns with an artificially fast respiratory rate, the small changes in impedance delay have a relatively large effect on the time relationship between heart rate and impedance curve. Therefore, from Figure 2 no definite conclusions can be drawn concerning this time relationship. It still can be seen that a fluctuation in heart rate occurs synchronously with artificial and spontaneous ventilation. The influence of artificial ventilation on heart rate is not obviously reversed as found by Yli-Hankala et al. (18). This might be ascribed to the less appropriate respiration measurement (impedance instead of tracheal pressure measurement), but might also be due to entrainment of the central respiratory activity by artificial ventilation. Entrainment of the central respiratory rhythm (as measured by phrenic nerve activity) by periodic lung inflation has been described in animals (20) and humans (21). This entrainment is only present with intact vagus nerves and is mediated via stretch receptors in the lung (HeringBreuer inflation reflex); thus, unsedated preterm infants may synchronize their inspiratory activity with the ventilator. Therefore, RSA in artificially ventilated infants may mimic normal RSA and be predominantly initiated by the central respiratory center. The amount of RSA in both spontaneously breathing as well as in ventilated infants gives information about the function of the parasympathetic autonomic nervous system. However, not only the gain of the system but also the strength of the perturbation determines the amount of RSA. It might be questioned whether the strength of the perturbation is the same in both spontaneously breathing and ventilated infants.

Artificial RSA has been reported in children with brain death (22) and in newborns with RDS (23) without giving information about the time relationship between heart rate and respiration. In brain death, the vagal input of the heart is assumed to be blocked because brain stem and medulla oblongata (containing the nucleus of the vagal nerve) are destroyed (22). The heart rate fluctuations synchronous with artificial ventilation, present in some infants with cerebral brain death, were ascribed to a peripheral (sympathetic) mechanism or survival of afferent vagal pathways in these infants. Äärima et al. (23) found ventilator-induced RSA in preterm newborns with RDS, as did we. In their study, the ventilator-induced RSA decreased with advancing age (d 1 to 5 after birth). However, no information was given about the rate of artificial ventilation. In addition, their RDS study group consisted of both spontaneously breathing and artificially ventilated preterm infants. In 

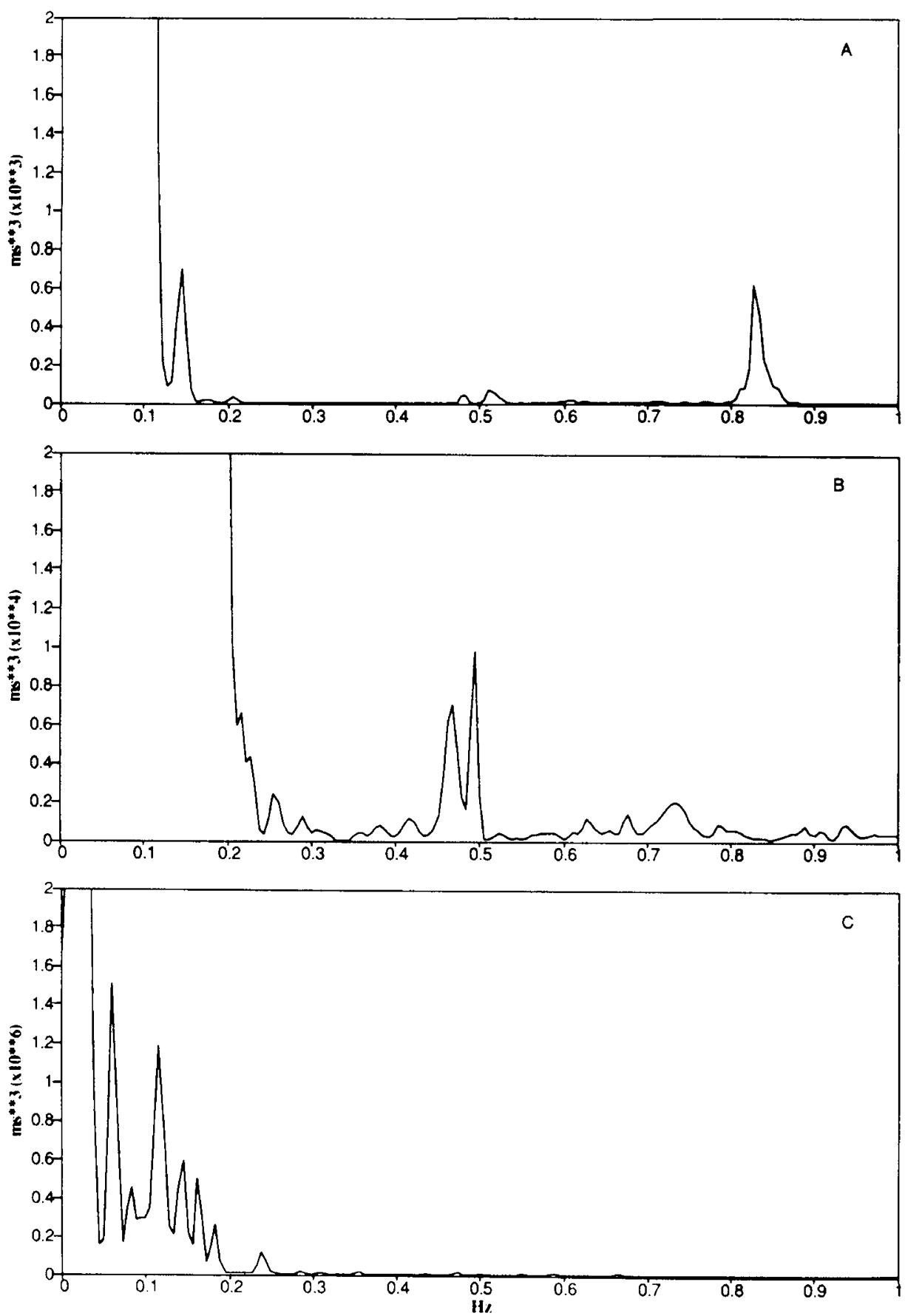

Figure 3. Influence of aVR on RSA. R-R interval power spectrum of patient 19 at the age of $12(A), 36(B)$, and $72(C) \mathrm{h}$ after birth, while ventilated at 0.83 , 0.50 , and $0.12 \mathrm{~Hz}$, respectively. The spectral peak corresponding to the aVR increases from $0.65 \times 10^{3}$ to $1.0 \times 10^{4}$ and $1.2 \times 10^{6} \mathrm{~ms}^{3}$, respectively.

our study, after correction for ventilation rate, gestational age, and R-R interval length, a small positive correlation between aRSA and age was found (Table 4).

To the best of our knowledge, the influence of aVR on HRV has not been studied before in human newborns. The influence of aVR on HRV was assessed by stepwise regression analysis to correct for the known influences of postnatal and gestational age, R-R interval length, and RDS severity $(1,2,8,23)$. In our study group of artificially ventilated preterm newborns, VLF and LF HRV were influenced by postnatal and gestational age as previously found for spontaneously breathing preterm newborns (Table 4). In contrast to spontaneously breathing infants, only a minor influence of mean R-R interval length, but a more obvious influence of RDS severity on VLF HRV, was found in ventilated infants. HF HRV was influenced by R-R interval length in both spontaneously breathing and ventilated infants. In the ventilated infants, no significant influence of postnatal age but a significant influence of aVR on HF HRV was found. This might be due to a very tight central coupling between inspiratory activity and cardiac activity or a difference in strength of the spontaneous and artificial perturbation.

The negative correlation between aVR and aRSA is partly due to the index used, because at lower aVR this aRSA index also includes VLF HRV. However, after exclusion of lower 
Table 4. Step-wise regression analysis of factors that influence $H R V$ in artificially ventilated and spontaneously breathing preterm newborns

\begin{tabular}{lcccc}
\hline & VLF & LF & HF & RSA $^{*}$ \\
\hline Artificial ventilation & & & & \\
R-R & & $0.01 \dagger$ & $0.19 \ddagger$ & $0.06 \ddagger$ \\
Age & $0.13 \ddagger$ & $0.20 \ddagger$ & & $0.01 \ddagger$ \\
GA & $0.08 \ddagger$ & $0.09 \ddagger$ & & $0.03 \ddagger$ \\
RDS & $0.11 \ddagger$ & $0.10 \ddagger$ & & \\
$\quad$ aVR & & $0.01 \ddagger$ & $0.04 \ddagger$ & $0.44 \ddagger$ \\
Spontaneous breathing & & & & \\
R-R & $0.20 \ddagger$ & $0.27 \ddagger$ & $0.16 \ddagger$ & $0.02 \ddagger$ \\
Age & $0.13 \ddagger$ & $0.17 \ddagger$ & $0.21 \ddagger$ & \\
GA & $0.02 \dagger$ & $0.04 \ddagger$ & & \\
VR & & & & $0.20 \ddagger$ \\
\hline
\end{tabular}

Results of stepwise regression analysis with HRV indices as dependent variables and $R-R$ interval length (R-R), postnatal age, GA, RDS severity, aVR, or median spontaneous ventilation rate (VR) as independent variables. The data from the spontaneously breathing infants were obtained from a former study (8). The significant partial correlation coefficients $\left(R^{2}\right)$ are shown. The influences found are all based on positive correlations, except for aVR, VR, and RDS. $p<0.05 . p<0.001$.

$*$ aRSA for artificially ventilated newborns.

$\dagger p<0.05$.

$\ddagger p<0.001$

aVR, a negative influence between aVR and aRSA was still found (Table 3 and Fig. 3). Higher aVR is accompanied by more severe RDS. Step-wise regression analysis revealed that RDS severity predominantly influenced VLF HRV, and aVR HF HRV. Thus, RDS severity is not likely to be the main cause for the negative correlation between aVR and aRSA. A more likely explanation is the occurrence of entrainment between baroreffex-related heart rate oscillations and RSA as found in spontaneously breathing adults (15) and newborns $(8,24)$. Entrainment between respiratory- and baroreflex-related oscillations occurs if the respiratory rate approaches $0.07 \mathrm{~Hz}$ in newborns. Therefore, RSA increases as respiratory rate decreases. Similarly, an increase in aRSA can be expected as aVR decreases. The strong influence of aVR on the amount of HF HRV indicates that in ventilated infants suffering from RDS the parasympathetic division of the autonomic nervous system is functioning adequately. However, the negative influence of RDS severity on LF HRV seems not to be due to artificial ventilation.

In summary, the negative correlation between aVR and aRSA is partly due to the aRSA index used and is not likely to be connected with severity of RDS. Artificial ventilation causes heart rate fluctuations that mimic spontaneous RSA. The heart rate fluctuations caused by artificial ventilation (aRSA) exhibit entrainment with baroreflex-related heart rate oscillations. This entrainment is comparable to the interaction between RSA and baroreflex-related oscillations in spontaneously breathing newborns and indicates that the parasympathetic nervous system is functioning quite adequately in preterms suffering from RDS.
In studies concerning the influence of RDS on HRV, it has been established that RDS predominantly attenuates LF HRV $(2,6,7)$. This could be confirmed by our study. Inasmuch as artificial ventilation especially influences HF HRV, the hitherto found attenuation of LF HRV by RDS is not due to artificial ventilation, but might be ascribed to poor regulation of blood pressure and temperature (25).

\section{REFERENCES}

1. Van Ravenswaaij-Arts CMA, Hopman JCW, Kollée LAA, Van Amen JPL, Stoelinga GBA, Van Geijn HP 1991 Influences on heart rate variability in spontaneously breathing preterm infants. Early Hum Dev 27:187-205

2. Van Ravenswaaij-Arts CMA, Hopman JCW, Kollée LAA, Van Amen JPL, Stoelinga GBA, Van Geijn HP 1991 The influence of respiratory distress syndrome on heart rate variability in very preterm infants. Early Hum Dev 27:207-221

3. Giddens DP, Kitney RI 1985 Neonatal heart rate variability and its relation to respiration. J Theor Biol 113:759-780

4. Dykes FD, Ahmann PA, Baldzer K, Carrigan TA, Kitney R, Giddens DP 1986 Breath amplitude modulation of heart rate variability in normal full term neonates. Pediatr Res 20:301-308

5. Akselrod S, Gordon D, Madwed JB, Snidman NC, Shannon DC, Cohen RJ 1985 Hemodynamic regulation: investigation by spectral analysis. Am J Physiol 249:H867--H875

6. Jenkins JG, Reid MMcC, McClure BG 1980 Study of heart rate variability in sick newborn infants. Acta Paediatr Scand 69:393-396

7. Korvenranta H, Kero P, Välimäki I 1983 Cardiovascular monitoring in infants with respiratory distress syndrome. Biol Neonate 44:138-145

8. Van Ravenswaaij-Arts CMA, Hopman JCW, Kollée LAA, Stoelinga GBA, Van Geijn HP 1994 Spectral analysis of heart rate variability in spontaneously breathing very preterm infants. Acta Paediatr 83:473 - 480

9. Nijhuis JG, Prechtl HFR, Martin CB, Bots RSGM 1982 Are there behavioural states in the human fetus? Early Hum Dev 6:177-195

10. Jenkins GM, Watts DG 1969 Spectral analysis and its applications. Holden-Day, San Francisco

11. Baldzer K, Dykes FD, Jones SA, Brogan M, Carrigan TA, Giddens DP 1989 Heart rate variability analysis in full-term infants: spectral indices for study of neonatal cardiorespiratory control. Pediatr Res 26:188-195

12. SAS Institute Inc. 1985 SAS User's Guide: Statistics, Version 5 Ed. SAS Institute Inc Cary, NC

13. Gaultier C, Praud JP, Canet E, Delaperche MF, D'Allest AM 1987 Paradoxical inward rib cage motion during rapid eye movement sleep in infants and young children. J Dev Physiol 9:391-397

14. Feldman JL, Ellenberger HH 1988 Central coordination of respiratory and cardiovascular control in mammals. Ann Rev Physiol 50:593-606

15. Hirsch JA, Bishop B 1981 Respiratory sinus arrhythmia in humans: how breathing pattern modulates heart rate. Am J Physiol 241:H620-H629

16. Kitney R, Linkens D, Selman A, McDonald A 1982 The interaction between heart rate and respiration: part II. Nonlinear analysis based on computer modelling. Automedica 4:141-153

17. Melcher A 1980 Carotid baroreflex heart rate control during the active and assisted breathing cycle in man. Acta Physiol Scand 108:165-171

18. Yli-Hankala A, Porkkala T, Kaukinen S, Häkkinen V 1991 Respiratory sinus arrhythmia is reversed during positive pressure ventilation. Acta Physiol Scand 141:399-407

19. Victorin L, Daily W, Olsson T 1970 Transthoracic impedance: III. Methodological studies in newborn infants. Acta Paediatr Scand [Suppl] 207:37-47

20. Muzzin S, Baconnier P, Benchetrit G 1992 Entrainment of respiratory rhythm by periodic lung inflation: effect of airflow rate and duration. Am J Physiol 263:R292R300

21. Graves C, Glass L, Laporta D, Meloche R, Grassino A 1986 Respiratory phase locking during mechanical ventilation in anesthetized human subjects. Am J Physiol 250:R902-R909

22. Kero P, Antila K, Ylitalo V, Välimäki I 1978 Decreased heart rate variation in decerebration syndrome: quantitative clinical criterion of brain death? Pediatrics 62:307-311

23. Äärimaa T, Oja R, Antila K, Välimäki I 1988 Interaction of heart rate and respiration in newborn babies. Pediatr Res 24:745-750

24. Hathorn MKS 1989 Respiratory modulation of heart rate in newborn infants. Early Hum Dev 20:81-99

25. Waldman S, Krauss AN, Auld PAM 1979 Baroreceptors in preterm infants: Their relationship to maturity and disease. Dev Med Child Neurol 21:714-722 